IdeAs

Idées d'Amériques

15 | 2020

Eau et gestion de l'eau dans les Amériques

\title{
Du mouvement féministe étudiant au mouvement social \#ChileDespertó
}

Camila Ponce

\section{OpenEdition}

1 Journals

Édition électronique

URL : http://journals.openedition.org/ideas/8396

DOI : $10.4000 /$ ideas.8396

ISSN : 1950-5701

Éditeur

Institut des Amériques

Référence électronique

Camila Ponce, "Du mouvement féministe étudiant au mouvement social \#ChileDespertó », IdeAs [En ligne], 15 | 2020, mis en ligne le 01 mars 2020, consulté le 25 mars 2020. URL : http:// journals.openedition.org/ideas/8396 ; DOI : https://doi.org/10.4000/ideas.8396

Ce document a été généré automatiquement le 25 mars 2020

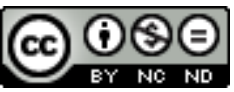

IdeAs - Idées d'Amériques est mis à disposition selon les termes de la licence Creative Commons Attribution - Pas d'Utilisation Commerciale - Pas de Modification 4.0 International. 


\title{
Du mouvement féministe étudiant au mouvement social \#ChileDespertó
}

\author{
Camila Ponce
}

1 Le mouvement féministe étudiant a occupé le devant de la scène au cours de l'année 2018 en devenant le plus important mouvement étudiant depuis celui de 2011 pour une éducation publique gratuite et de qualité. Ces mobilisations ont contribué à la convergence de revendications historiques telles que celles liées aux droits en matière de sexualité et de procréation n'ayant encore jamais réussi à véritablement s'inscrire à l'agenda gouvernemental (Baeza C. et Ponce C., sous presse). À la différence des autres mouvements féministes latino-américains ou internationaux, celui de 2018 a, de plus, surgi du cœur des institutions universitaires (Follegatti L., 2018 ; Ponce C., 2020) à partir d'assemblées dites féministes et une précédente politisation des étudiants chiliens. L'élément déclencheur d'un tel mouvement a été une série de plaintes pour harcèlement sexuel au sein et en dehors des salles de classe ayant conduit les élèves à s'organiser et se mobiliser afin de mettre en place des protocoles et de dénoncer toute violence subie au quotidien.

2 Ce mouvement présente plusieurs caractéristiques spécifiques et notamment le fait qu'il se compose: d'activistes séparatistes (Miranda L. et B. Roque, 2019) prônant l'organisation d'assemblées et d'actions menées uniquement par des femmes; d'activistes davantage soucieux de la rédaction de protocoles communs avec les différentes instances universitaires; ou encore d'autres groupes militants favorables à plus d'intersectionnalité au sein du mouvement et à l'intégration d'autres dimensions, ethniques, migratoires, de socioéconomiques ou générationnelles. Ce mouvement a également été rejoint par d'autres organisations déjà présentes sur la scène sociale à l'image des groupes en faveur de la dépénalisation inconditionnelle de l'avortement ou contre le féminicide et les stéréotypes autour du corps de la femme.

3 Le mouvement ou « soulèvement » social chilien (estallido social) a ainsi vu le jour le 18 octobre 2019 lorsque les lycéens de Santiago ont décidé de ne pas payer le transport 
public pour protester contre la hausse de 30 pesos du prix du ticket de métro (voir article de Faure et Maillet dans ce dossier). Parti d'un appel lancé via un compte Instagram à ne pas payer leurs titres de transport pendant la semaine du 14 octobre, ce mouvement a su conglomérer l'ensemble des revendications présentes lors des précédentes manifestations.

\section{Le mai féministe de 2018}

4 Le mouvement féministe de 2019 présente des spécificités qui le distinguent tout en le rapprochant d'autres mouvements féministes. Nous nous proposons ici de développer trois de ces aspects centraux : son caractère global, son origine universitaire et son caractère performatif.

5 Il convient tout d'abord de souligner la dimension mondiale de ce mouvement. Contrairement à certaines investigations qui assimilent ce mouvement à une troisième vague féministe ${ }^{1}$ (Oyarzún K., 2018 ; Valdés T., 2018 ; Reyes-Housholder C. et B. Roque, 2019), il semble préférable de ne pas négliger d'autres vagues de revendications comme celle en faveur du droit à l'avortement distincte de la deuxième vague féministe au niveau mondial ${ }^{2}$ et apparue au Chili depuis la période post-dictatoriale et à diverses reprises au cours des années 1990 et 2000 (Baeza C. et Ponce C., sous presse). La troisième vague féministe représenterait, dès lors, pour certains auteurs (Biswas A., 2004) une nouvelle génération cherchant à célébrer et revendiquer la diversité culturelle, sociale, religieuse, ethnique et sexuelle.

Le mouvement féministe de 2018 relève donc plutôt d'une quatrième vague féministe mondiale en faveur de la justice sociale et contre le harcèlement sexuel et les violences faites aux femmes (Chamberlain P., 2017 ; Ponce C., 2020) et se caractérise par le recours aux nouvelles technologies (Cochrane K., 2013) et à de nouveaux modes de revendication tels que l'expression corporelle et la performance. C'est ainsi que le mai féministe se relie au mouvement \#NiUnaMenos (PasUneDeMoins) apparu en Argentine en 2016 et ayant eu depuis des répercussions aux quatre coins du continent sudaméricain (Pérou, Chili et Uruguay). Il fait écho également aux rassemblements de femmes contre Bolsonaro au Brésil (\#EleNão) et aux grandes manifestations des "foulards verts" de 2018 en Argentine en faveur d'un droit à l'avortement sûr et gratuit déjà réclamé par les collectifs féministes en 2005 (Larrondo M. et C. Ponce, 2019).

7 Comme indiqué précédemment, et à la différence du célèbre \#MeToo et de son équivalent français \#BalanceTonPorc qui se sont propagés grâce aux réseaux sociaux, le mouvement chilien a d'abord vu le jour dans les universités. Le printemps féministe est un mouvement qui s'est développé et a gagné un large soutien populaire sur la base des accusations faites à l'encontre de professeurs d'universités chiliennes, et est donc étroitement lié à la violence de genre et au harcèlement sexuel. Les revendications qu'il incarne renvoient aux problèmes de violence patriarcale au sein des universités ainsi qu'aux enseignements sexistes et au langage utilisé par les professeurs et les élèves. Il s'agit donc d'un mouvement qui, d'une certaine façon, rassemble les revendications des précédentes mobilisations étudiantes jusqu'à remettre en question le système éducatif chilien dans son ensemble en l'accusant de discriminer les élèves en fonction de leur niveau socioéconomique et de ne pas leur fournir les outils nécessaires à un épanouissement équitable. 
8 Mais ce mouvement présente également d'autres caractéristiques, comme le fait de s'inscrire dans un courant historique plus large créé depuis plusieurs décennies par le féminisme chilien. Il s'articule avec d'autres mouvements ayant pour but de dénoncer et d'en finir avec le féminicide et d'autres formes d'oppression et de violences économiques, sexuelles, politiques et sociales qu'endurent tous les jours les femmes des classes ouvrières, migrantes ou mapuches.

9 La troisième caractéristique du mouvement féministe étudiant chilien repose sur le rôle donné au corps et à la sphère privée. Le corps devient ici un instrument supplémentaire de protestation capable de revendiquer son droit à ne pas être un simple objet de jouissance masculine ou de consommation et à lutter contre la société patriarcale et les violences faites aux femmes. La sphère privée devient, dès lors, elle aussi centrale à travers la désacralisation du corps féminin et la fin des tabous qui existent autour des vulves des femmes que l'on n'hésite plus à représenter grandes et colorées (Ponce C., 2020). Le fait de tout mettre au grand jour, d'exprimer ou de montrer tout ce qui dérange s'inscrit dans cette même démarche consistant à tout déballer, des abus commis par de grands universitaires respectés jusqu'aux petites culottes tachées de sang que l'on exhibe en signe de provocation.

Comme répertoire d'action, les étudiantes ont choisi d'avoir recours aux performances comme, par exemple, lorsqu'elles se sont moquées des concours Miss Reef ${ }^{3}$ en défilant avec des queues de cheval en référence à l'artiste emblématique Pedro Lemebel et aux Juments de l'Apocalypse ${ }^{4}$. D'autres types de performances cherchent également à rendre présentes, à l'aide de textes et d'images, des femmes victimes de féminicides ou ayant été assassinées pendant la dictature.

11 Ce mouvement, bien que massif au sein de l'espace public où il est capable de mobiliser des milliers de personnes - non seulement à travers des processions traditionnelles, mais également dans le cadre de performances ou de spectacles de rue - et même après être parvenu à occuper des dizaines d'institutions dans tout le pays au cours de l'année $2018^{5}$, a en effet peiné à traduire ses revendications en un agenda concret et s'est progressivement affaibli au cours du second semestre 2018. Les revendications contre le patriarcat et les pratiques machistes ont fini par s'étioler au fil de protocoles sur l'égalité entre les genres et de procédures judiciaires contre des professeurs et des élèves.

12 Avant le soulèvement social de 2019, a eu lieu, le 8 mars 2019, une grève de femmes du monde entier ayant eu des répercussions dans tout le Chili et ayant donné lieu à la plus importante manifestation féministe de l'histoire chilienne avec plus de 800000 personnes défilant dans les rues des principales villes du pays (El Mostrador, 2019).

\section{Le soulèvement ou le grand mouvement social du \#ChileDespertó}

Ce mouvement est né du cri et du malaise des lycéens, lesquels ont toujours joué un rôle central dans les principales mobilisations de la période post-dictatoriale. Ils ont, en effet, constitué le principal moteur de changement du système éducatif chilien. Les mouvements lycéens trouvent, pour la plupart, leur origine dans des revendications à l'encontre du transport public. Lors de chacune de ces mobilisations étudiantes, les gouvernements en place sont parvenus à les déstabiliser en criminalisant la 
contestation sociale et en discréditant le maillon le plus faible et le plus violent de ces mouvements, à savoir l'émeutier cagoulé (el encapuchado). La société rejetant massivement la violence et condamnant tout acte de vandalisme, les encapuchados sont ainsi devenus le talon d'Achille des mouvements étudiants et au fur et à mesure des violences, des débordements et de l'œuvre d'agents infiltrés dans les protestations, l'espoir d'un changement possible s'est peu à peu évanoui.

Mais les choses semblent toutefois avoir désormais changé et la figure de l'encapuchado, bien qu'encore criminalisée par les médias et le gouvernement, est dorénavant revendiquée et mise en valeur par une grande partie des manifestants. L'encapuchado n'est plus uniquement perçu comme un sujet violent ou un exclu en marge de la société, mais constitue celui qui se place en première ligne dans la lutte en cours et permet au reste des manifestants de continuer de se faire entendre dans la rue. Les encapuchados incarnent ainsi, à présent, ceux qui, bien que camouflés derrières leurs cagoules, s'emploient à transformer la société chilienne.

Le mouvement actuel qui cherche à déconstruire le modèle néolibéral chilien n'est pas qu'étudiant et mobilise plusieurs secteurs de la société, transversalement en termes de tranche d'âge, de genre, de ville de résidence ou de niveau socioéconomique. Des mobilisations aussi massives et violentes sont nouvelles dans le Chili démocratique et le conflit ne semble pas devoir être résolu à court terme, les institutions en place ne semblant pas en mesure ou disposées à apporter de solution ni à interagir avec l'ensemble des acteurs de la société civile (Ponce C., 2019).

Les activistes présents dans les rues cherchent avant tout à en finir avec les enclaves autoritaires héritées de la dictature, ce qui se traduit essentiellement par des demandes telles que la rédaction d'une nouvelle constitution, la fin des fonds de pension (AFP) et le droit à des salaires dignes et un système de santé plus juste. Des revendications comme la fin des zones sacrifiées (zonas de sacrificio) et la nationalisation de l'eau sont également soulevées, bien qu'avec moins de véhémence et de consensus. Il en va de même en ce qui concerne certaines exigences de nature féministe, lesquelles, bien que présentes lors des manifestations à travers les groupes de femmes mobilisées (notamment les foulards verts portés par des centaines d'entre elles), n'étaient pas toujours visibles et pleinement représentées par les pancartes brandies par les manifestants.

17 C'est ainsi qu'au fil des semaines, le besoin s'est fait sentir d'incorporer la dimension féministe à cette vague de revendications sociales, notamment du fait que les viols et les abus de la part de la police et des militaires étaient devenus récurrents dans un tel contexte de répression (voir article d'Aguilera dans ce dossier). Dans le même temps, la propre ministre de la Femme et à l'Égalité entre les sexes, Isabel Plá, s'est révélée incapable d'assurer la fin des violences à caractère sexiste dans le cadre du mouvement social et préférait détourner l'attention en consacrant ses tweets sur les policières ayant été victimes de violences (El desconcierto, 2019). Un tel comportement explique pourquoi les manifestants ont rapidement exigé sa démission (La Tercera, 2019), comme en attestent les messages inscrits sur de nombreuses pancartes. À l'occasion de plusieurs performances, des femmes portant des foulards verts se sont également mobilisées devant son ministère afin de réclamer sa démission et ainsi que celle du président Piñera.

18 Le projet de transformation sociale d'influence féministe établi en 2018 (Follegati L., 2018) a, dès lors, refait surface à l'occasion de ce soulèvement social. Les performances 
féministes ont progressivement pris une place cruciale au sein du mouvement en organisant, en plus des journées classiques de manifestation spécialement consacrées à la dénonciation du patriarcat et des violences contre les femmes, différentes actions visant à dénoncer la torture et la mort de femmes sous la dictature comme pendant le mouvement en cours. C'est dans un tel contexte qu'est apparue la performance de Las Tesis dont le but était de remobiliser un mouvement social qui montrait ses premiers signes d'essoufflement.

19 Las Tesis est un collectif interdisciplinaire de femmes de Valparaiso qui s'emploie, à partir de performances et de techniques visuelles, à exposer dans la rue et dans l'espace public des théories féministes inspirées des idées de Silvia Federici et de Rita Segato (Las Tesis, 2019). Comme elles-mêmes l'expliquent, leur « intervention est le résultat d'un travail de recherche que le collectif mène depuis le début de l'année sur le viol. Cette chanson est devenue une performance à partir de l'appel lancé par différents artistes de Valparaiso de créer des interventions artistiques dans la rue " (Las Tesis, 2019). La performance permet, dès lors, de donner un nouveau visage au patriarcat. Ciblant jusqu'en 2018 des individus ayant un nom et un prénom -ce qui affaiblissait les revendications, il désigne désormais des institutions en général : le violeur - comme l'indique le manifeste - c'est la police ; c'est la justice ; bref, c'est l'État.

Le mouvement s'est ainsi reconfiguré autour de cette chanson et de cette chorégraphie contre la violence à caractère sexiste devenue du jour au lendemain un véritable manifeste féministe au niveau international. Un chant qui a permis aux activistes, hommes et femmes, de donner plus de visibilité à leurs mobilisations et à leurs revendications grâce notamment aux milliers de militantes en ayant spontanément reproduit la chorégraphie dans plus de 100 villes du monde. D'Istanbul à Mexico, ces représentations intersectionnelles ont aussi bien mobilisé des femmes jeunes ou du troisième âge, des ouvrières comme des professeures, des étudiantes et des cadres ou encore des femmes handicapées.

21 Cette performance a, finalement, contribué à faire comprendre que le projet d'un nouveau Chili passe par les femmes et les mouvements féministes, mais aussi que néolibéralisme et patriarcat vont de pair. La modalité de la performance scénographique est, par ailleurs, parvenue à attirer de nombreuses femmes fatiguées des violences sexistes et de la manière dont les manifestations étaient réprimées par l'État, en les invitant à danser dans les rues de toutes les villes chiliennes et du reste du monde. Beaucoup d'entre elles reconnaissent, en effet, que cette danse leur a permis de se libérer, de se fédérer et de ne plus avoir peur. Les femmes ont pris conscience qu'elles sont capables de se dépasser elles-mêmes et entendent jouer un rôle fondamental dans l'actuelle transformation de la société chilienne. 


\section{BIBLIOGRAPHIE}

Baeza, Cecilia et Ponce, Camila, «De la "Loi des 3 causes" au plus grand mouvement féministe de l'histoire du Chili : dynamiques, actrices, et nouvelles subjectivités », Problèmes d'Amérique latine, (sous presse).

Biswas, Andrea, « La tercera ola feminista: cuando la diversidad, las particularidades y la diferencia son lo que cuenta », Casa del Tiempo, vol.6, n68, 2004, p.65-70.

Chamberlain, Prudence, The feminist fourth wave: Affective temporality. Houndmills, UK, Palgrave Macmillan, 2017.

Cochrane, Kira, All the rebel women: The rise of the fourth wave of feminism, Londres, Guardian Books, 2013.

De Beauvoir, Simone, El segundo sexo, Buenos Aires, Ediciones Siglo Veinte, 1987.

El Desconcierto, « Interpelan a ministra Plá tras mensaje de apoyo a Carabineras: Acusan que contrasta con su silencio por casos de violencia sexual », El Desconcierto, 5 de noviembre 2019, https://urlz.fr/bBhG, page consultée le 09/03/2020

El Mostrador, « Desbordante movilización feminista concentra a varios cientos de miles de personas en Santiago y regiones ", El Mostrador, 9 de marzo de 2019, https://urlz.fr/bAQN, page consultée le 09/03/2020

Follegati, Luna, « El feminismo se ha vuelto una necesidad: movimiento estudiantil y organización feminista (2000-2017) », Anales de la Universidad de Chile, 2018, n¹4.

Kirkwood, Julieta Ser política en Chile. Las feministas y los partidos, Santiago, FLACSO, 1986. Larrondo, Marina y Camila, Ponce, « Activismos feministas jóvenes en América Latina. Dimensiones y perspectivas conceptuales ", in Larrondo, M. \& C. Ponce (eds.), Activismos feministas jóvenes: emergencias, actrices y luchas en América Latina. Buenos Aires. CLACSO, 2019, p.21-38.

La Tercera (2019). « Realizan protesta fuera del Ministerio de la Mujer exigiendo la renuncia de Isabel Plá ». La Tercera, 29 de noviembre de 2019, https://urlz.fr/bBpZ, page consultée le 09/03/2020

Las Tesis (2019). « Manifiesto del Colectivo Las Tesis: La culpa no era mía, ni dónde estaba ni cómo vestía », The Clinic, 10 de diciembre de 2019, https://urlz.fr/bBqr, page consultée le $09 / 03 / 2020$

Miranda, Lucía y Beatriz, Roque, « El mayo estudiantil feminista de 2018 en la Pontificia Universidad Católica de Chile. "La revolución es Feminista" », in Larrondo, Marina \& Camila Ponce (eds.): Activismos feministas jóvenes: emergencias, actrices y luchas en América Latina. Buenos Aires, CLACSO, 2019, p.59-78.

Oyarzún, Kemy en Hugo Guzmán, « Esta tercera ola es la lucha por las identidades », El Siglo, 29 de mayo del 2018, https://urlz.fr/bAQC, page consultée le 09/03/2020

Ponce, Camila, « El movimiento estudiantil feminista de 2018: Continuidades y rupturas entre feminismos y olas globales », Revista Izquierdas, $\mathrm{n}^{\circ} 49,2020$, p. 1554-1570.

Ponce, Camila, « El estallido de Chile: un movimiento que exige cambios estructurales », Open Democracy, 23 de octubre del 2019, https://urlz.fr/bAQw, page consultée le 09/03/2020 
Valdés, Teresa en Paulina Sepúlveda, « Feminismo chileno vive su revolución más importante en 40 años », La Tercera, 20 de mayo 2018, https://urlz.fr/bAQy, page consultée le 09/03/2020

Reyes-Housholder, Catherine y Beatriz, Roque, « Chile 2018: desafíos al poder de género desde la calle hasta La Moneda », Revista de ciencia política, vol.39, n², 2019, p.191-216.

\section{NOTES}

1. Les deux premières étant les suffragettes du début du xxe siècle (1930-1950) dont la lutte se centrait sur le droit de vote des femmes, et les mouvements féministes rassemblés sous la devise « la démocratie dans le pays et à la maison » durant la dictature (Kirkwood, 1986)

2. La deuxième vague de féminisme serait celle cherchant à différencier les éléments biologisants liés au fait d'être une femme et le genre à l'image de la célèbre phrase de Simone de Beauvoir : «On ne nait pas femme : on le devient » (De Beauvoir S., 1987).

3. Concours organisés en été pour élire les plus belles fesses et au cours desquels les candidates ne montrent pas leur visage.

4. Voir article complémentaire : Ponce, Camila (2020). "El movimiento estudiantil feminista de 2018: Continuidades y rupturas entre feminismos y olas globales”. Revista Izquierdas, n49, p. 1554-1570

5. Il s'agit ici d'institutions universitaires de type religieux, régional, entrepreneurial, public ou apostolique, ainsi que de lycées publics et privés.

\section{AUTEUR}

\section{CAMILA PONCE}

Camila Ponce est affiliée au CISJU - Université Catholique Silva Henríquez

camila.poncelara@gmail.com 